\title{
Polyclonal origin of hormone-producing cell populations evaluated as a direct in situ demonstration in EGFP/BALB/C chimeric mice
}

\author{
De-Fu Ma, Katsuko Sudo', Hideo Tezuka², Tetsuo Kondo, Tadao Nakazawa, Dong-Feng Niu, \\ Tomonori Kawasaki, Kunio Mochizuki, Tetsu Yamane and Ryohei Katoh \\ Department of Pathology, University of Yamanashi, 1110 Shimokato, Chuo Yamanashi 409-3898, Japan \\ ${ }^{1}$ Tokyo Medical University Animal Research Center, Tokyo Medical University, Tokyo 160-8402, Japan \\ ${ }^{2}$ Center for Life Science Research, University of Yamanashi, Yamanashi 409-3898, Japan \\ (Correspondence should be addressed to R Katoh; Email: rkatoh@yamanashi.ac.jp; defuma2008@hotmail.com)
}

\begin{abstract}
We report the first demonstration of the embryonal patch patterns of endocrine organs and the polyclonality of hormone-producing cell populations using chimeric mice produced by aggregation of C57BL/6-Tg(CAG-EGFP)C14Y01-FM131Osb transgenic mice and BALB/C mice. Confocal laser scanning microscopy (CLSM) analysis for enhanced green fluorescent protein (EGFP) and immunohistochemistry with anti-EGFP antibody revealed that all endocrine organs of chimeric mice had a mosaic appearance of EGFP-positive patches and EGFP-negative patches. The patches composed of EGFP-positive cells were distinctive in their size and shape. The pituitary patches were large and irregular, representing a geographical pattern. In contrast, parathyroid, pancreatic islet, and adrenal medulla patches were small and demarcated, representing an island-like pattern. Thyroid follicles and adrenal cortex cords showed a mixture of monophenotypia and polyphenotypia, indicating
\end{abstract}

polyclonal embryonic origin. Furthermore, we studied the tissue clonality of hormone-producing cell populations in the pituitary, thyroid, and pancreatic islets using a combination method of CLSM for EGFP and immunohistochemistry for hormones. All the pituitary cell populations of $\mathrm{GH}$, prolactin, $\mathrm{TSH}, \mathrm{FSH}, \mathrm{LH}$, and $\mathrm{ACTH}$, the calcitonin-producing cell population in the thyroid, and the insulin- and glucagonproducing cell populations in pancreatic islets had mosaic patterns in EGFP expression in the chimeric mice, suggesting polyclonal embryonic origin. In conclusion, the different patch patterns of the endocrine organs could contribute to the understanding of embryonic development and organization of endocrine organs. Furthermore, we clearly demonstrate that all hormone-producing cell populations are of polyclonal embryonic origin, derived from more than two progenitor cells.

Journal of Endocrinology (2010) 207, 17-25

\section{Introduction}

A cellular marker that can be used to identify a subset of germline cells is necessary for examining the clonal architecture of normal tissue (Novelli et al. 2003). Analyzing chimeric animals may potentially provide resolution of this issue (Tarkowski 1961, Thompson et al. 1990). A persistent difficulty for such studies has been the lack of suitable histological markers for the different animal strains. Many markers have been used in chimeras to differentiate the parental strains, including pigmentary differences such as coat color and retinal pigment, histochemical differences, and DNA markers (Rossant et al. 1983, Schmidt et al. 1985). The problem with these markers is that they are not easily detectable in histological sections of most organs.

Since Chalfie et al. (1994) reported that the green fluorescent protein (GFP), derived from the jellyfish Aequorea victoria, can serve as a useful marker of gene expression without substrate loading or other pretreatment, GFP has opened the door for the use of intact cells and organisms as experimental systems (Misteli \& Spector 1997). In 1997, Okabe et al. (1997) produced 'green mice' using enhanced GFP (EGFP) and reported that all the organs of the transgenic mouse lines were uniformly green with a few exceptions of hair and red blood cells, which facilitates the use of these animals in clonality research of most organs. We reported in our previous study that, although the level of EGFP expression varied among organs and tissues of the 'green mice,' EGFP is diffusely and strongly expressed in all of the endocrine organs including pituitary, thyroid, parathyroid, adrenal cortex, adrenal medulla, and the pancreatic islets, which suggests that 'green mice' could facilitate clonality research of endocrine organs (Ma et al. 2010).

Endocrine tissues with a high level of hormonal responsiveness are often nodular or undergo nodular hyperplasia or neoplastic formation. Appropriate interpretation of clonality 
Table 1 Development of aggregation chimeras

Number of aggregates
transferred

128

\begin{tabular}{c}
$\begin{array}{c}\text { Number of } \\
\text { recipients }\end{array}$ \\
\hline 8
\end{tabular}

Number of
live newborns

90

$\frac{\begin{array}{c}\text { Number of } \\ \text { chimeras }\end{array}}{70}$

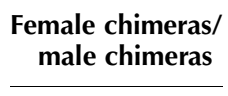

$38 / 32$ information in endocrine tissues should be very useful because the pathogenesis of many endocrine tumors is unclear, and the histological characteristics that remain central to establishing prognosis in most neoplasias are of limited value in endocrine tumors (Levy 2001). We hope that a better understanding of endocrine organ development will eventually contribute to the development of novel therapies for treating endocrine diseases, especially endocrine tumors. To the best of our knowledge, there has been little investigation into embryonal patch architecture and embryonic development of endocrine organs.

To systematically and comprehensively clarify embryonal patch patterns and embryonic development of endocrine organs, we produced chimeric mice by aggregating BALB/C mouse and 'green mouse' embryos. We analyzed the EGFP expression in tissue sections from endocrine organs with immunohistochemistry using anti-EGFP antibody and confocal laser scanning microscopy (CLSM) for EGFP under excitation light. Moreover, we evaluated the clonality of individual hormone-producing cell populations, including $\mathrm{GH}$, prolactin (PRL), TSH, FSH, LH, and ACTH cells in pituitary, C cells in thyroid, and the insulin-producing $\beta$-cells and glucagonsecreting $\alpha$-cells in pancreatic islets with immunofluorescence using specific antibodies directed against the hormone combined with CLSM for EGFP under excitation light.

\section{Materials and Methods}

Mice

The animal experimental procedure and care of laboratory animals followed the Guidelines for Animal Experiments of the Yamanashi University. The two types of embryos used for the construction of aggregation chimeras were from crosses of $\mathrm{BALB} / \mathrm{C}$ mice and C57BL/6-Tg(CAG-EGFP)C14-Y01FM131Osb transgenic mice (EGFP mice). The BALB/C mice were white. The EGFP mice from a transgenic line with an EGFP cDNA under the control of a chicken $\beta$-actin promoter and cytomegalovirus enhancer were black and were kindly gifted by Prof. Okabe of Osaka University. Chimeric mice were produced by aggregation of embryos as described previously (Deltour et al. 1991). Briefly, females of EGFP mice and BALB/C mice were superovulated by an i.p. injection of $5 \mathrm{IU}$ of pregnant mare serum, followed $46 \mathrm{~h}$ later by i.p. injection of 5 IU of human chorionic gonadotropin. They were then placed with males and checked for vaginal plugs the next morning (day 1). We collected eight-cell stage embryos on day 3 of pregnancy. After removing the zona pellucida, pairs of embryos, one of each type, were aggregated in microdrops and cultured overnight. Embryos that formed chimeras and developed into single blastocysts were transferred into the uterine horns of day 3 pseudopregnant Institute of Cancer Research recipient females.

\section{Histological sections}

Endocrine organs, which included pituitary, thyroid, parathyroid, adrenal, and pancreas, of chimeric mice and control mice (BALB/C and EGFP mice) were obtained by dissection, fixed in $8 \%$ buffered paraformaldehyde in PBS for $2 \mathrm{~h}$ at room temperature, and embedded in paraffin. Two micrometer thick sections were cut and mounted on glass slides pretreated with poly-L-lysine for immunostaining and CLSM.

\section{Reagents and antibodies}

Polyclonal rabbit anti-human glucagon (N1541, ready to use), polyclonal guinea pig anti-human insulin (N1542, ready to use), rabbit antibody against human TSH (L1842, ready to use), rabbit antibody against human FSH (L1810, ready to

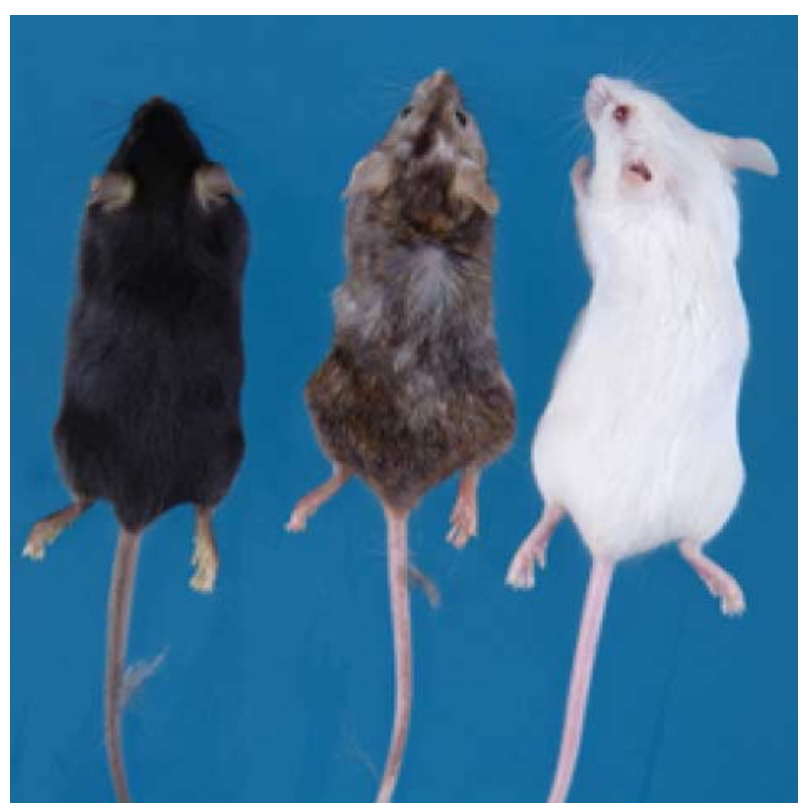

Figure 1 External findings of the 'EGFP mouse' (left), chimeric mouse (middle), and BALB/C mouse (right). The chimeric mouse presents with a chimeric coat of black from the 'green mouse' and white from the $\mathrm{BABL} / \mathrm{C}$ mouse. Full colour version of this figure available via http://dx.doi.org/10.1677/JOE-10-0184. 
use), rabbit antibody against human LH (L1827, ready to use), rabbit antibody against human ACTH (N1531, 1:1000), polyclonal rabbit anti-human calcitonin (N1552, ready to use), HRP-linked rabbit secondary antibody (P0217, 1:100), and the rhodamine-conjugated swine immunoglobulin to rabbit immunoglobulin (R156, 1:20 in PBS) were purchased from DAKO (Carpinteria, CA, USA). Rhodamineconjugated donkey immunoglobulin to mouse immunoglobulin (AP192R, 1:20 in PBS) was purchased from Chemicon International (Temecula, CA, USA). Rabbit anti-A victoria GFP (A11122, 1:1000) was obtained from Molecular Probes (Eugene, OR, USA). Rabbit polyclonal anti-human GH (11181, 1:100) was ordered from Progen Biotechnik (Heidelberg, Germany). Rabbit anti-mouse PRL (1:3000) was obtained from National Hormone and Peptide Program (Torrance, CA, USA). All of the anti-human hormone antibodies are cross-reactive with mouse hormones.

\section{Immunoperoxidase method for EGFP}

The immunohistochemistry procedure for EGFP has been described elsewhere (Walter et al. 2000). Briefly, after removing paraffin wax in three changes of xylene and a series of ethanols, endogenous peroxidase was blocked by immersing the sections in $3 \% \mathrm{H}_{2} \mathrm{O}_{2}$ for $10 \mathrm{~min}$. The sections were incubated for $1 \mathrm{~h}$ at room temperature with primary antibody of EGFP. Tissue sections were then reacted with HRP-linked rabbit secondary antibody for $40 \mathrm{~min}$ at room temperature. Visualization of EGFP was performed with diaminobenzidine for $6 \mathrm{~min}$. After counterstaining with hematoxylin for $10 \mathrm{~s}$, the sections were dehydrated in a series of graded ethanols, cleared in three changes of xylene, and coverslipped for light microscopy.

\section{CLSM for EGFP}

Two micrometer thick sections were deparaffinized in xylene and rehydrated in a series of ethanols. Deparaffinized sections were counterstained with TOPRO-3 (Invitrogen) at a concentration of 1:500 in PBS for $1 \mathrm{~h}$ at room temperature. After counterstaining, the sections were coverslipped for light microscopy. We performed differential interference microscopy using a confocal laser scanning microscope (FV1000; Olympus, Tokyo, Japan).

\section{Immunofluorescence method for hormones}

After removing paraffin wax in three changes of xylene and a series of ethanols, endogenous peroxidase was blocked by immersing the sections in $3 \% \mathrm{H}_{2} \mathrm{O}_{2}$ for $10 \mathrm{~min}$. The sections
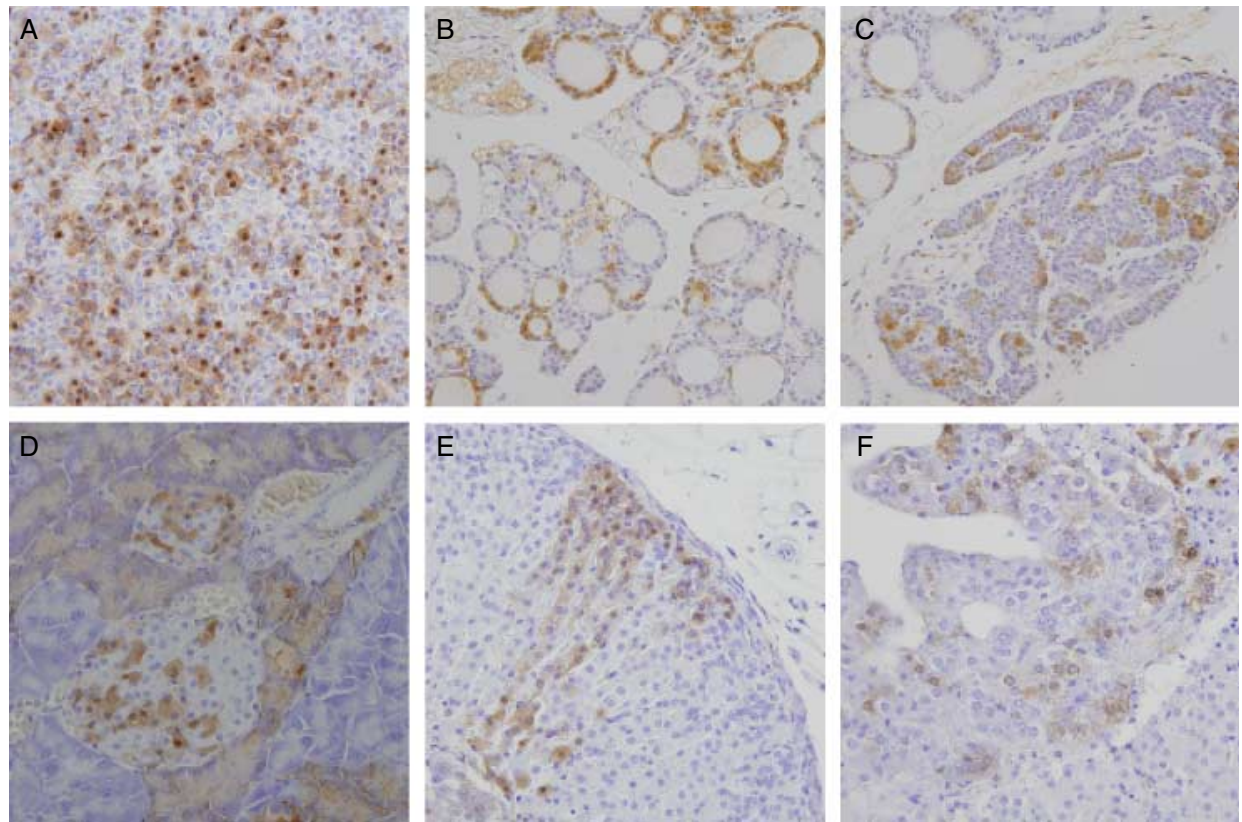

Figure 2 Immunohistochemical findings of EGFP in endocrine organs. Endocrine cells in endocrine organs (A-F) show mosaic patch patterns in EGFP expression. The patches composed of EGFP-positive cells are different in size and shape among the endocrine organs: geographical pattern in pituitary (A), island-like pattern in parathyroid (C), island-like pattern in pancreatic islet (D), and island-like pattern in adrenal medulla (F). Thyroid follicles have three patterns of EGFP expression: follicles composed of EGFP-positive cells, follicles composed of EGFP-negative cells, and follicles composed of both EGFP-positive and -negative cells (B). Similarly, we found three cord phenotypes in the adrenal cortex: monophenotypic EGFP-positive cords, monophenotypic EGFP-negative cords, and polyphenotypic cords (E). EGFP, enhanced green fluorescent protein. Magnification: $400 \times$. Full colour version of this figure available via http://dx.doi.org/10.1677/JOE-10-0184. 
were incubated for $1 \mathrm{~h}$ at room temperature with a specific primary antibody directed against the hormone-producing cells. Tissue sections were then reacted with the secondary antibody of swine anti-rabbit rhodamine or donkey antimouse rhodamine for $1 \mathrm{~h}$ at room temperature. Then, the sections were coverslipped for light microscopy. We used a confocal laser scanning microscope to perform differential interference microscopy.

\section{Results}

\section{Production of chimeric mice}

We transferred 128 aggregated embryos into 8 foster mothers and obtained 90 newborns. Of the 90 newborn mice, 70 (38 female mice and 32 male mice) were regarded as chimeric mice according to their chimeric coat (Table 1). The chimeric mice presented with a chimeric coat of black from the EGFP mice and white from the BALB/C mice, which indicated that the progenies of the amalgamation were dizygotic mosaic animals (Fig. 1).

All organ tissues from the chimeric mice were analyzed at postnatal (PN) week $1(n=10), \mathrm{PN}$ week $2(n=10), \mathrm{PN}$ week $3(n=10)$, PN week $4(n=10)$, PN week $12(n=10)$, PN week $26(n=10)$, and PN week $52(n=10)$. Immunohistochemistry for EGFP and CLSM for EGFP under excitation light revealed that a dual population of cells (EGFP-positive cells and EGFP-negative cells) existed as we expected in the tissues of chimeric mice, and that the discrimination between contiguous EGFP-positive and -negative patches and between individual cells was quite distinct. There was no difference in the EGFP expression pattern among any of the male/female chimeric mice at any experiment week (from PN week 1 to $\mathrm{PN}$ week 52).

Embryonal patch patterns in the endocrine organs of chimeric mice

In the present research, we measured the areas of the EGFP-positive parts and the total areas of the endocrine sections of chimeric mice at any experiment week (from PN week 1 to PN week 52) using Scion Image. There was no difference in the contribution of the EGFP-positive cells among any of the male/female chimeric mice at any experiment week (data not shown). We used sections of endocrine tissues from EGFP mice as a positive control and those from the BALB/C mice as a negative control (data not shown).

EGFP immunohistochemistry and CLSM for EGFP under excitation light revealed that the pituitary tissues in chimeric mice displayed mosaic patches of EGFP-positive and -negative cells. The patches composed of EGFP-positive cells were large and appeared in geographical patterns (Figs $2 \mathrm{~A}$ and $3 \mathrm{~A}$ ). In contrast, the patches of EGFP-positive cells observed in
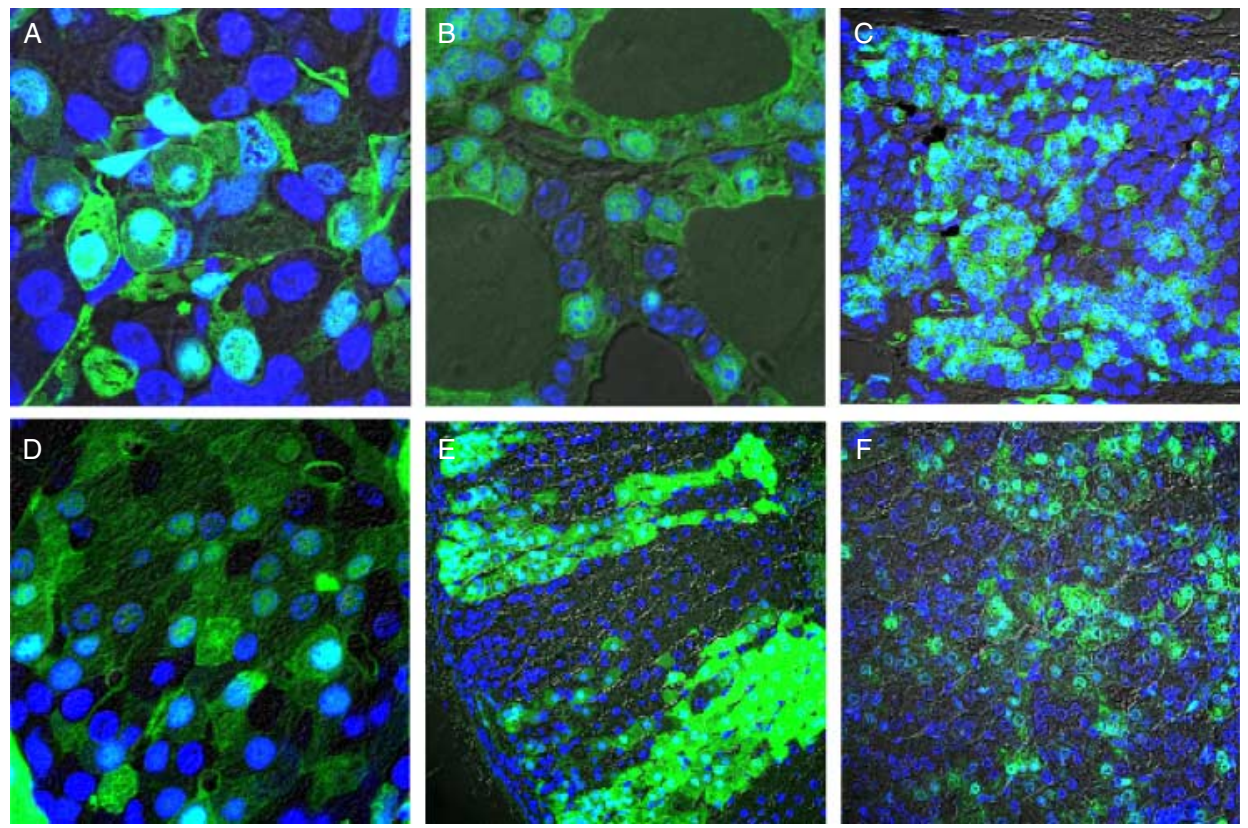

Figure 3 CLSM findings of EGFP under excitation light in endocrine organs. The mosaic patch patterns in EGFP expression are identical to those observed by immunohistochemistry in endocrine organs such as pituitary $(A)$, thyroid $(B)$, parathyroid $(C)$, pancreatic islet $(D)$, adrenal cortex $(E)$, and adrenal medulla $(F)$. EGFP, enhanced green fluorescent protein; CLSM, confocal laser scanning microscopy. Magnification: $1200 \times(A, B$, and D) and $600 \times(C)$ and $400 \times(E$ and F). Full colour version of this figure available via http://dx.doi.org/10.1677/JOE-10-0184. 

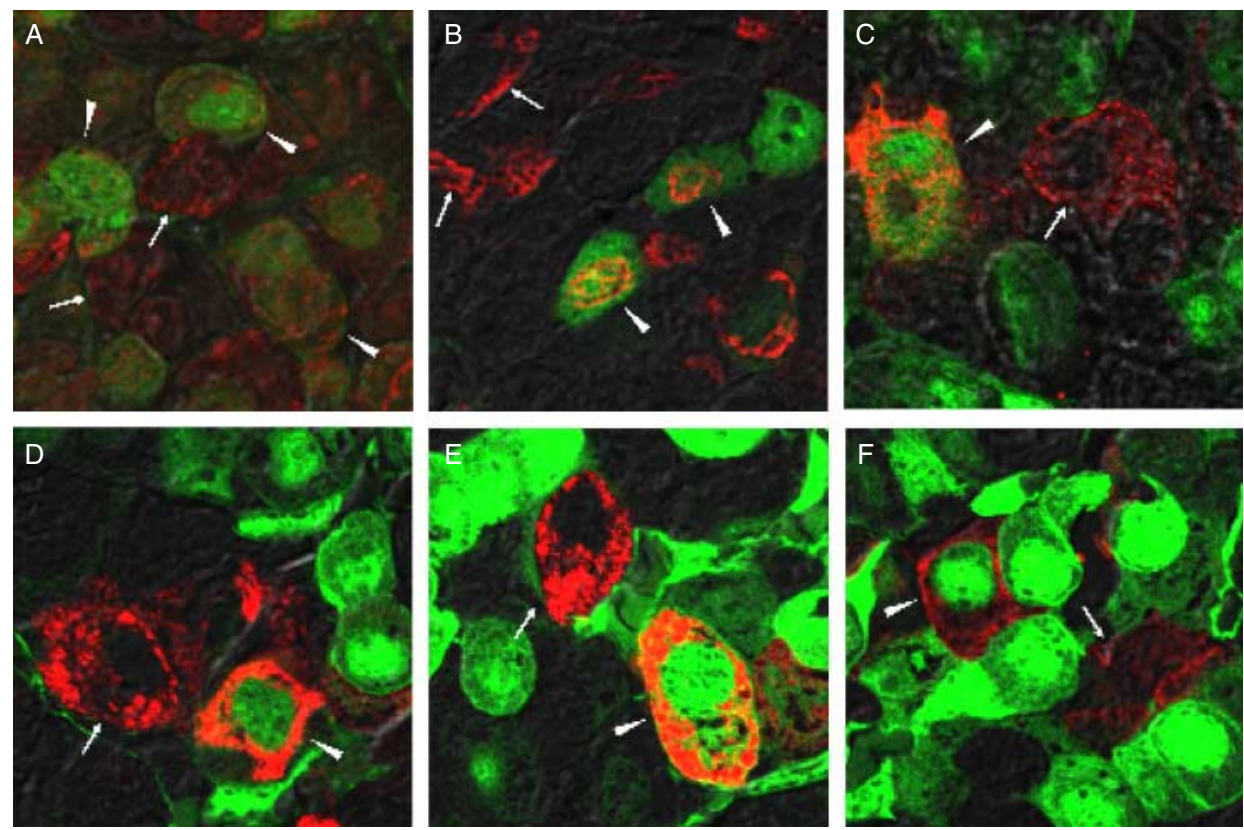

Figure 4 Merged images of CLSM for pituitary hormones (rhodamine: red) and EGFP (fluorescence: green). All hormone-producing cell populations (A, GH cells; B, PRL cells; C, TSH cells; D, FSH cells; E, LH cells; and F, ACTH cells) in the pituitary are composed of EGFP-positive cells (white arrowheads) and EGFP-negative cells (white arrows). EGFP, enhanced green fluorescent protein; CLSM, confocal laser scanning microscopy. Magnification: $1200 \times(\mathrm{A}-\mathrm{F})$. Full colour version of this figure available via http://dx.doi.org/10.1677/JOE-10-0184.

the parathyroid (Figs 2C and 3C), pancreatic islets (Figs 2D and $3 \mathrm{D}$ ), and adrenal medulla (Figs $2 \mathrm{~F}$ and $3 \mathrm{~F}$ ) were small in size and appeared in island-like patterns. Thyroid follicles had three patterns of EGFP expression: follicles composed of EGFP-positive cells, follicles composed of EGFP-negative cells, and follicles composed of both EGFP-positive and -negative cells (Figs 2B and 3B). Similarly, we found three cord phenotypes in the adrenal cortex: monophenotypic EGFP-positive cords, monophenotypic EGFP-negative cords, and polyphenotypic cords (Figs 2E and 3E). Distribution of the three phenotypic thyroid follicles and adrenal cortex cords appeared to be non-random, and some large aggregations of single phenotypic follicles or cords were observed in certain areas of thyroid tissue and adrenal cortex.

Clonality of hormone-producing cell populations in the pituitary, thyroid, and pancreatic islets

In the present study, we studied the clonality of hormoneproducing cell populations in the pituitary, thyroid, and pancreatic islets. Using computer modeling, the images obtained through immunofluorescence for a specific hormone (rhodamine: red) were merged with those obtained through CLSM for EGFP under excitation light (fluorescence: green).

In the pituitary, we found that hormone-producing cell populations, including GH, PRL, TSH, FSH, LH, and
ACTH, were heterogeneous in EGFP expression (Fig. 4A-F) in spite of the wide variety of cell numbers within hormoneproducing cell populations, suggesting polyclonal embryonic origin. Calcitonin-producing cell populations (C cells) in the thyroid were also composed of both EGFP-positive cells and EGFP-negative cells derived from more than two progenitor cells (Fig. 5). We observed similar results in the pancreatic islets: both the insulin-producing cell populations ( $\beta$-cells) and the glucagon-producing cell populations ( $\boldsymbol{\alpha}$-cells) were heterogeneous in their EGFP expression, representing polyclonal embryonic origin (Fig. 6A and B).

\section{Discussion}

We previously reported that EGFP is distinctly and diffusely expressed in the secretarial cells, especially endocrine cells such as pituitary hormone-producing cells, thyroid follicular cells, parathyroid cells, adrenal cells, and islet cells of the pancreas in the EGFP mice. This indicates that it may be possible to use 'EGFP mice' for determining the tissue clonality of endocrine organs in chimeric mice (Ma et al. 2010). In our present research, we produced chimeric mice by aggregation of BALB/C mice and EGFP mice to observe the patch patterns of endocrine organs in situ. This is the first use of EGFP whose expression does not need substrate loading or any other pretreatment as a histological marker for 


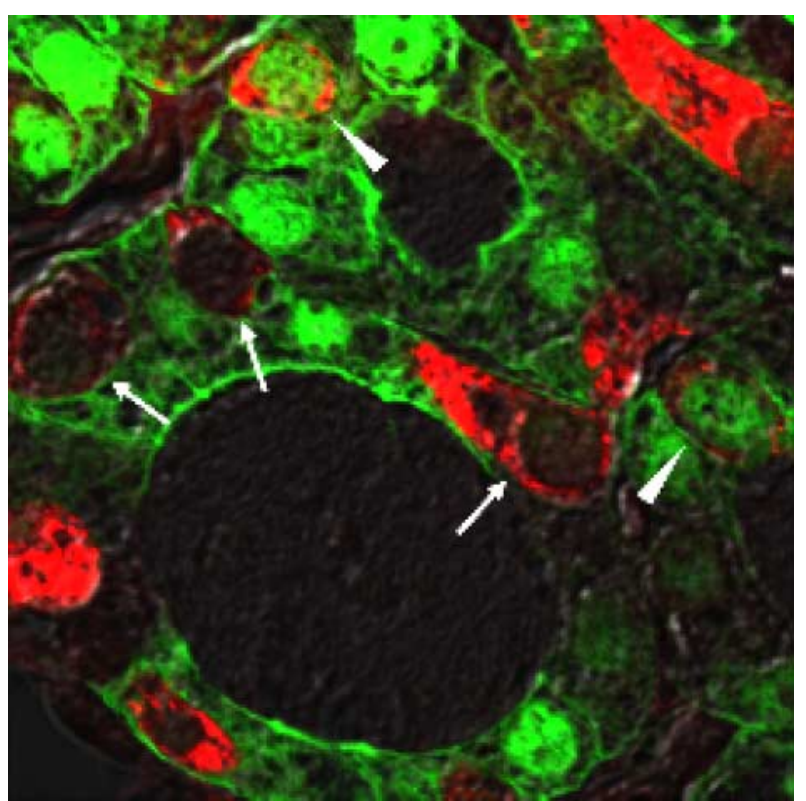

Figure 5 Merged images of CLSM for calcitonin (rhodamine: red) and EGFP (fluorescence: green) in thyroid. Calcitonin-immunopositive cells (C cells) in the thyroid are composed of EGFP-positive cells (white arrowheads) and EGFP-negative cells (white arrows). EGFP, enhanced green fluorescent protein; CLSM, confocal laser scanning microscopy. Magnification: $1800 \times$. Full colour version of this figure available via http://dx.doi.org/10.1677/JOE-10-0184.

studying embryonal patch patterns of endocrine organs. Ideally, a marker used to demonstrate variegation that is dependent on genetic polymorphism should be 1) cell autonomous in gene expression and not transferred between cells, 2) cell limited, i.e. not secreted into the extracellular environment, 3) developmentally neutral so that its presence does not produce a bias in the proportions of the two cellular phenotypes or an adverse effect on the development as a whole, 4) stable throughout embryogenesis and adult life, so that the relative proportions of the two cell types are not temporally variable, and 5) easily demonstrable in histological sections (McLaren 1976). Immunohistochemistry for EGFP and CLSM for EGFP under excitation light in the present study clearly demonstrated cellular expression of EGFP in the tissues of the chimeric mice we produced, which suggests that EGFP meets these requirements and, therefore, could be regarded as a suitable histological marker for studies on tissue clonality.

The potential value of using chimeric animals to assess the cell lineages during embryogenesis was appreciated when the first mammalian chimera was reported in 1961 (Tarkowski 1963). From then on, the investigations of the clonality of some organs such as determination of monoclonality of gastric glands and intestinal crypts were reported using chimeric mice (Ponder et al. 1985, Nomura et al. 1998). However, little is known about the embryonal patch patterns of endocrine organs and tissue clonality of hormone-producing cell populations because of a lack of suitable histological markers. In our present research, we systematically and comprehensively investigated the embryonal patch patterns of endocrine organs and the clonality of hormone-producing cell populations for the first time.
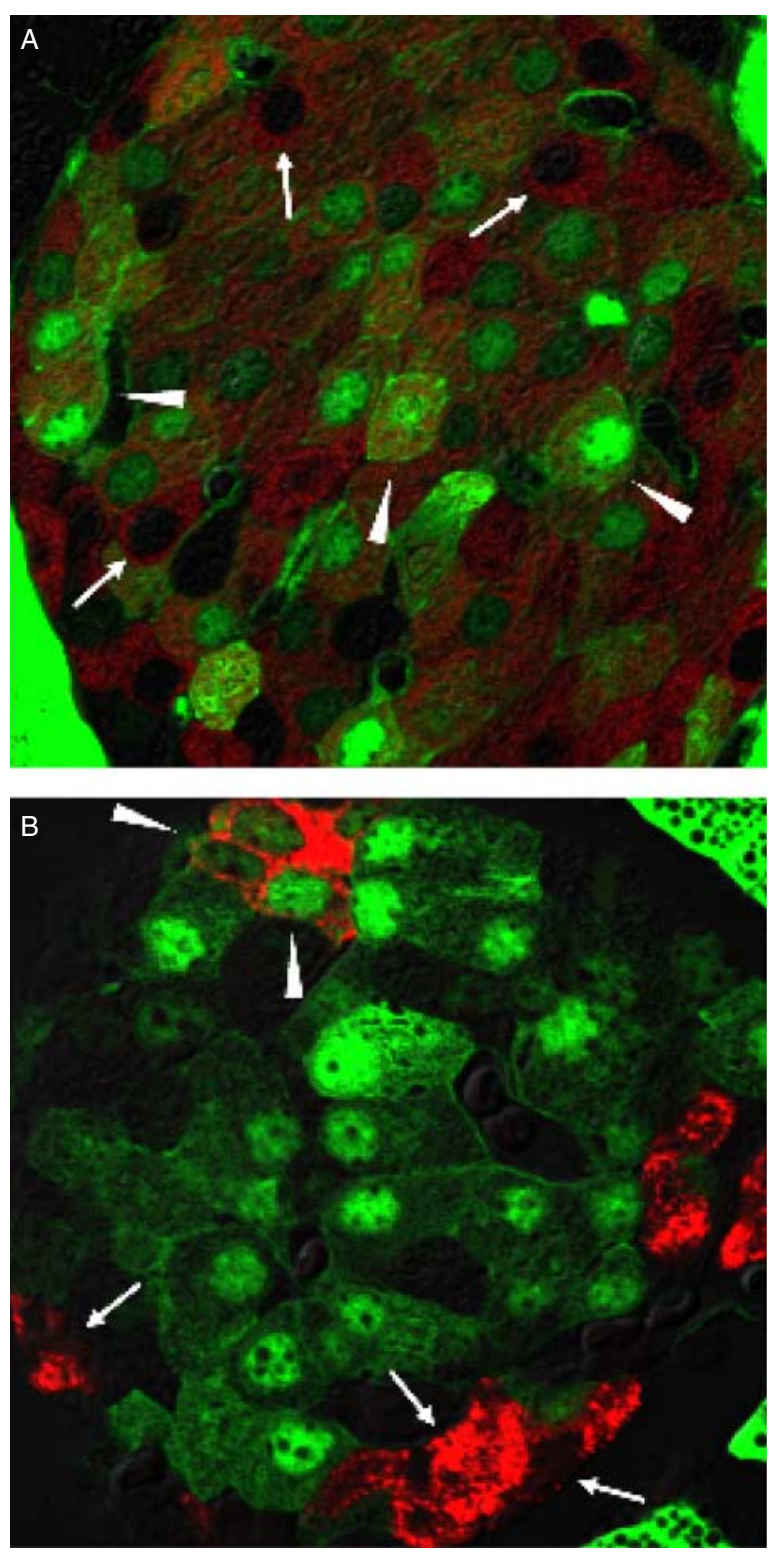

Figure 6 Merged images of CLSM for insulin or glucagon (rhodamine: red) and EGFP (fluorescence: green) in pancreatic islets. (A) Insulin-immunopositive cells ( $\beta$-cells) in islets are composed of EGFP-positive cells (white arrowheads) and EGFPnegative cells (white arrows). (B) Glucagon-secreting cells ( $\alpha$-cells) in islets are heterogeneous with both EGFP-positive cells (white arrowheads) and EGFP-negative cells (white arrows). EGFP, enhanced green fluorescent protein; CLSM, confocal laser scanning microscopy. Magnification: $1200 \times(\mathrm{A}$ and B). Full colour version of this figure available via http://dx.doi.org/10.1677/JOE-10-0184. 
The endocrine organs of different chimeric mice presented similar mosaic patterns, which suggested that the pattern appears to be conserved, while the mosaic pattern in different endocrine organs was different, suggesting that the pattern is regulated (Khokha et al. 1994).

Early in pituitary development, when the primordium is located at the midline of the anterior neural ridge and within Rathke's pouch, the cells appear uniform. The initial, apparently uniform, precursor cells proliferate and differentiate into six different cell types: GH, PRL, TSH, FSH, LH, and ACTH cells. No studies on the clonality architecture of individual hormone-producing cell population of the pituitary have been reported so far. We demonstrated that each of the hormone-producing cell populations recognized by immunofluorescence using specific antibodies directed against the hormone is composed of both EGFP-positive cells and EGFP-negative cells. Our results, therefore, suggest that each hormone-producing cell population derives from more than two progenitor cells in despite of that the number of individual hormone-producing cell population is very different: GH and PRL cell populations are very large in number, whereas TSH, FSH, LH, and ACTH cell populations are very small in number. Mulchaheym \& Jaffe (1988) reported that, in addition to the classical somatotrophs and lactotrophs, the fetal human pituitary contains an additional cell type that secretes both PRL and GH, and concluded that the dual hormone-secreting cells may represent a common progenitor of these classical cell types. More recently, some investigators found a small population of progenitor cells $(<0 \cdot 05 \%)$ in the adult pituitary gland and produced 'pituispheres' using these progenitor cells. They reported that all of the pituitary hormone cell types were found in the 'pituispheres'. Furthermore, different endocrine cell types could be found within single spheres (Chen et al. 2005, Fauquier et al. 2008). Considering these in vitro results, it is reasonable to suggest that there are more than two progenitor cells, and a single progenitor cell gives rise, in vivo, to one or all of the precursors of hormone-producing cell populations in the pituitary (Fig. 7).

There have been two studies investigating the clonal architecture of thyroid follicles. In both the X-linked enzyme glucose-6-phosphate dehydrogenase (G6PD) mosaic mice and the Sardinian females heterozygous for the G6PD Mediterranean mutation, thyroid follicles showed a mixture of mono- and polyphenotypia, consistent with a polyclonal origin (Thomas et al. 1988, Novelli et al. 2003). Our present study using the chimeric mice we produced demonstrates polyclonal origin of thyroid follicles and supports those results. Moreover, distribution of the three types of thyroid follicles appears to be non-random, forming large patches, suggesting that a certain amount of coherent clonal growth occurs during proliferation to produce a relatively large patch size for thyroid tissues. Jovanovic et al. (2003) determined the embryonal patch size of the human thyroid gland by microdissection of normal thyroid specimens. They reported that the monoclonal patch size of normal human thyroid

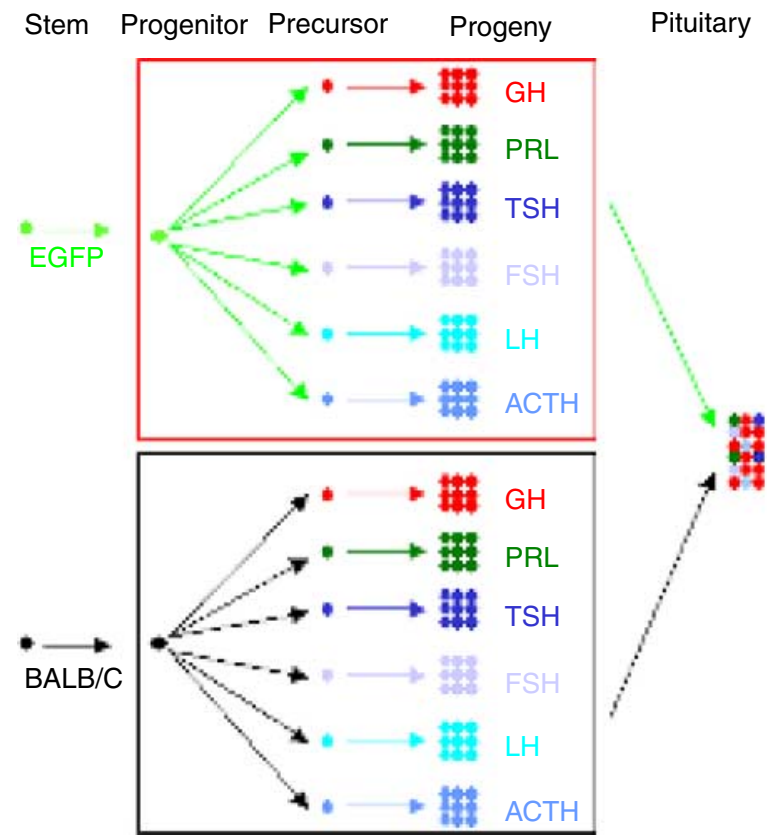

Figure 7 Schematic representation of the possible expansion of the cells from which the hormone-producing cells of the pituitary derive. There are two or more progenitor cells, and a single progenitor cell gives rise to one or all of the precursors of hormoneproducing cell populations.

tissue is between 48 and $128 \mathrm{~mm}^{2}$, and suggested that normal thyroid epithelium is organized into large stem cell-derived monoclonal patches. It is generally known that thyroid $\mathrm{C}$ cells are derived from neuroectoderm and are different to follicular cells arising from foregut endoderm in embryonic origin. We could find no reported studies on the clonality of thyroid C cells. Our study clearly demonstrated that the thyroid C cells are polyclonal in embryonic origin, which may contribute to the understanding of follicle development.

Pancreatic islets, with their characteristic architecture of central insulin-producing cells and non-insulin-producing cells in the periphery, do not form until near the end of gestation. It is generally believed that islet formation occurs by the aggregation of pre-existing cells. However, there has been little investigation into the clonal architecture of the insulinproducing $\beta$-cell and glucagon-secreting $\alpha$-cell. A previous study analyzing aggregation mouse chimeras reported that the insulin-producing $\beta$-cell in most islets of the chimeric mice derived from both of the aggregated embryos, which suggested that the $\beta$-cells of each islet resulted from more than two progenitor cells (Deltour et al. 1991). In our present study, we demonstrated that not only the $\beta$-cell (insulincontaining cells) population but also the $\alpha$-cell (glucagoncontaining cells) population, the number of which is very small, was heterogeneous in EGFP expression, suggesting that the insulin-producing $\beta$-cell and glucagon-secreting $\alpha$-cell of each islet resulted from more than two progenitor cells, that is 
of polyclonal origin. Moreover, the patches of EGFP-positive cells within islets were very small, composed of small clusters of very few cells. Hellerstrom \& Swenne (1985) reported that the number of pancreatic endocrine cells increases strikingly between days 13 and 18 of fetal development, while the mitotic activity is very modest in this cell population. Moreover, the number of the DNA-synthesizing cells around the growing fetal islets is more than that in the islets themselves (Hellerstrom \& Swenne 1985). Altogether, these studies suggest that fetal islet growth mainly results from recruitment of precursors located in the vicinity of the islets. In contrast, large patches of endocrine cells with a single genotype observed in the pituitary imply a quite different pattern of growth, i.e. the in situ proliferation of endocrine cells (Dewey \& Mintz 1978).

The adrenal gland consists of cortex and medulla, which are different in embryonic origin, character, and function. We found few published studies on the embryonal patch pattern of the adrenal cortex, which reported that the adrenal cortex presented a mosaic expression of the class I MHC histological marker in mosaic animals (Iannaccone \& Weinberg 1987, Morley et al. 1996). In our study, we found three phenotypes of adrenal cortex cords: monophenotypic EGFP-positive cords, monophenotypic EGFP-negative cords, and polyphenotypic cords, indicating that adrenal cortex cords are of polyclonal embryonic origin. Iannaccone et al. (2003) reported that the adrenal cortex can regenerate after extirpation of the organ, which leaves only a remnant of the zona glomerulosa. Along with the results of our present study, this suggests that there may be a common histogenesis of the three functionally distinct zones, the zona glomerulosa, the zona fasciculata, and the zona reticularis. In addition, we also demonstrated the mosaic expression of EGFP showing an island-like patch pattern in the adrenal medulla of chimeric mice for the first time. The difference in patch patterns between the adrenal cortex and adrenal medulla suggests that the patch pattern is regulated, while the different mosaic patch patterns within the cortex and medulla may be associated with the development of different functional tissues, which is helpful for constructing hypotheses of organ growth (Khokha et al. 1994).

In conclusion, the different patch patterns among endocrine tissues observed in situ in the chimeric mice we produced may be associated with their functional characteristics and contribute to the understanding of embryonic development and organization of endocrine organs. Furthermore, we clearly demonstrated that all of the hormone-producing cell populations are of polyclonal embryonic origin derived from more than two progenitor cells regardless of the cell number of each hormone-producing cell population.

\section{Declaration of interest}

The authors declare that there is no conflict of interest that could be perceived as prejudicing the impartiality of the research reported.

\section{Funding}

This research did not receive any specific grant from any funding agency in the public, commercial or not-for-profit sector.

\section{Acknowledgements}

The authors wish to acknowledge Sankyo Labo Service Corporation of Japan for technical assistance. We also thank Prof. Tetsuro Kobayashi for his excellent advice.

\section{References}

Chalfie M, Tu Y, Euskirchen G, Ward WW \& Prasher DC 1994 Green fluorescent protein as a marker for gene expression. Science 263 802-805. (doi:10.1126/science.8303295)

Chen J, Hersmus N, Van Duppen V, Caesens P, Denef C \& Vankelecom H 2005 The adult pituitary contains a cell population displaying stem/ progenitor cell and early embryonic characteristics. Endocrinology 146 3985-3998. (doi:10.1210/en.2005-0185)

Deltour L, Leduque P, Paldi A, Ripoche MA, Dubois P \& Jami J 1991 Polyclonal origin of pancreatic islets in aggregation mouse chimaeras. Development 112 1115-1121.

Dewey MJ \& Mintz B 1978 Genetic control of cell-type-specific levels of mouse $\beta$-galactosidase. Developmental Biology 66 560-563. (doi:10.1016/ 0012-1606(78)90259-2)

Fauquier T, Rizzoti K, Dattani M, Lovell-Badge R \& Robinson IC 2008 SOX2-expressing progenitor cells generate all of the major cell types in the adult mouse pituitary gland. PNAS 105 2907-2912. (doi:10.1073/pnas. 0707886105)

Hellerstrom C \& Swenne I 1985 Growth pattern of pancreatic islets in animals. In The Diabetic Pancreas, pp 53-79. Eds BW Volk \& ER Arquilla. New York: Plenum Publishing Corporation.

Iannaccone PM \& Weinberg WC 1987 The histogenesis of the rat adrenal cortex: a study based on histologic analysis of mosaic pattern in chimeras. Journal of Experimental Zoology 243 217-223. (doi:10.1002/jez. 1402430207)

Iannaccone P, Morley S, Skimina T, Mullins J \& Landini G 2003 Cord-like mosaic patches in the adrenal cortex are fractal: implications for growth and development. FASEB Journal 17 41-43. (doi:10.1096/fj.02-0451fje)

Jovanovic L, Delahunt B, McIver B, Eberhardt NL \& Grebe SK 2003 Thyroid gland clonality revisited: the embryonal patch size of the normal human thyroid gland is very large, suggesting X-chromosome inactivation tumor clonality studies of thyroid tumors have to be interpreted with caution. Journal of Clinical Endocrinology and Metabolism 88 3284-3291. (doi:10.1210/ jc.2002-021552)

Khokha MK, Landini G \& Iannaccone PM 1994 Fractal geometry in rat chimeras demonstrates that a repetitive cell division program may generate liver parenchyma. Developmental Biology 165 545-555. (doi:10.1006/dbio. 1994.1274)

Levy A 2001 Monoclonality of endocrine tumours: what does it mean? Trends in Endocrinology and Metabolism 12 301-307. (doi:10.1016/S10432760(01)00434-9)

Ma DF, Tezuka H, Kondo T, Sudo K, Niu DF, Nakazawa T, Kawasaki T, Yamane T, Nakamura N \& Katoh R 2010 Differential tissue expression of enhanced green fluorescent protein in 'green mice'. Histology and Histopathology 25 749-754.

McLaren A 1976 Mammalian Chimaeras. Cambridge: Cambridge University Press. Misteli T \& Spector DL 1997 Applications of the green fluorescent protein in cell biology and biotechnology. Nature Biotechnology 15 961-964. (doi:10.1038/nbt1097-961)

Morley SD, Viard I, Chung BC, Ikeda Y, Parker KL \& Mullins JJ 1996 Variegated expression of a mouse steroid 21-hydroxylase/ $\beta$-galactosidase transgene suggests centripetal migration of adrenocortical cells. Molecular Endocrinology 10 585-598. (doi:10.1210/me.10.5.585) 
Mulchaheym JJ \& Jaffe RB 1988 Detection of a potential progenitor cell in the human fetal pituitary that secretes both growth hormone and prolactin. Journal of Clinical Endocrinology and Metabolism 66 24-32. (doi:10.1210/ jcem-66-1-24)

Nomura S, Esumi H, Job C \& Tan SS 1998 Lineage and clonal development of gastric glands. Developmental Biology 204 124-135. (doi:10.1006/dbio.1998.9055)

Novelli M, Cossu A, Oukrif D, Quaglia A, Lakhani S, Poulsom R, Sasieni P, Carta P, Contini M, Pasca A et al. 2003 X-inactivation patch size in human female tissue confounds the assessment of tumor clonality. PNAS $\mathbf{1 0 0}$ 3311-3314. (doi:10.1073/pnas.0437825100)

Okabe M, Ikawa M, Kominami K, Nakanishi T \& Nishimune Y 1997 'Green mice' as a source of ubiquitous green cells. FEBS Letters 407 313-319. (doi:10.1016/S0014-5793(97)00313-X)

Ponder BA, Schmidt GH, Wilkinson MM, Wood MJ, Monk M \& Reid A 1985 Derivation of mouse intestinal crypts from single progenitor cells. Nature 313 689-691. (doi:10.1038/313689a0)

Rossant J, Vijh M, Siracusa LD \& Chapman VM 1983 Identification of embryonic cell lineages in histological sections of $M$. musculus in-equilibrium $M$. caroli chimaeras. Journal of Embryology and Experimental Morphology 73 179-191.

Schmidt GH, Wilkinson MM \& Ponder BA 1985 Cell migration pathway in the intestinal epithelium: an in situ marker system using mouse aggregation chimeras. Cell 40 425-429. (doi:10.1016/0092-8674(85)90156-4)
Tarkowski AK 1961 Mouse chimaeras developed from fused eggs. Nature 190 857-860. (doi:10.1038/190857a0)

Tarkowski AK 1963 Studies on mouse chimeras developed from eggs fused in vitro. National Cancer Institute Monographs 11 51-71.

Thomas GA, Williams D \& Williams ED 1988 The demonstration of tissue clonality by X-linked enzyme histochemistry. Journal of Pathology 155 101-108. (doi:10.1002/path.1711550205)

Thompson EM, Fleming KA, Evans DJ, Fundele R, Surani MA \& Wright NA 1990 Gastric endocrine cells share a clonal origin with other gut cell lineages. Development 110 447-481.

Walter I, Fleischmann M, Klein D, Muller M, Salmons B, Gunzburg WH, Renner M \& Gelbman W 2000 Rapid and sensitive detection of enhanced green fluorescent protein expression in paraffin sections by confocal laser scanning microscopy. Histochemical Journal 32 99-103. (doi:10.1023/ A:1004014211408)

Received in final form 1 July 2010

Accepted 12 July 2010

Made available online as an Accepted Preprint

13 July 2010 